\title{
Stage IIIB Appendix Carcinoma AJCC v7
}

National Cancer Institute

\section{Source}

National Cancer Institute. Stage IIIB Appendix Carcinoma A/CC v7. NCI Thesaurus. Code C87804.

Stage IIIB includes: (T3, N1, M0); (T4, N1, M0). T3: Tumor invades through the muscularis propria into subserosa or into mesoappendix. T4: Tumor penetrates visceral peritoneum including mucinous peritoneal tumor within the right lower quadrant and/or directly invades other organs or structures. N1: Metastasis in 1-3 regional lymph nodes. M0: No distant metastasis. (AJCC 7th ed.) 